\title{
Using Competing Bacterial Communication to Disassemble Biofilms
}

\author{
Daniel P. Martins \\ Telecommunication Software \\ \& Systems Group (TSSG) \\ Waterford Institute of \\ Technology (WIT) \\ Waterford, Ireland. \\ dpmartins@tssg.org
}

\author{
Michael T. Barros \\ Telecommunication Software \\ \& Systems Group (TSSG) \\ Waterford Institute of \\ Technology (WIT) \\ Waterford, Ireland. \\ mbarros@tssg.org
}

\author{
Sasitharan \\ Balasubramaniam \\ Nano Communications Center \\ (NCC), Department of \\ Electronics and \\ Communication Engineering \\ Tampere University of \\ Technology (TUT) \\ Tampere, Finland. \\ sasi.bala@tut.fi
}

\begin{abstract}
In recent years, bacterial infections have become a major public health concern due to their ability to cooperate between single and multiple species resisting to various forms of treatments (e.g., antibiotics). One form of protection is through biofilms, where the bacteria produce a protective medium known as the Extracellular Polymeric Substances $(E P S)$. Researchers are pursuing new multi-disciplinary approaches to treating and kerb the evolving process of these infections through the biofilms, to lower the humans' antibiotic dependence that can result in the so-called "superbugs". Although various solutions have been proposed to break biofilms, they are based on applying drugs or using nanoparticles. In this paper, we propose an alternative approach, where bacteria will cooperate and surround the biofilms to consume the nutrients. By hijacking the nutrients in the environment and blocking the flow from reaching the biofilms, this will lead to starvation, forcing them to break their structure. Preliminary simulations show that a small action radius of quorum sensing molecules is needed to maximise bacteria attraction to a particular location and create the protective wall. Therefore, this formation is capable of speeds up biofilm dispersal process by two hours.
\end{abstract}

\section{CCS Concepts}

-Applied computing $\rightarrow$ Systems biology; Telecommunications; Computational biology; Systems biology;

\section{Keywords}

Biofilm; Synthetically engineered bacteria; Molecular communication.

Permission to make digital or hard copies of all or part of this work for personal or classroom use is granted without fee provided that copies are not made or distributed for profit or commercial advantage and that copies bear this notice and the full citation on the first page. Copyrights for components of this work owned by others than ACM must be honored. Abstracting with credit is permitted. To copy otherwise, or republish, to post on servers or to redistribute to lists, requires prior specific permission and/or a fee. Request permissions from permissions@ acm.org.

NANOCOM'16, September 28-30, 2016, New York, NY, USA

(C) 2016 ACM. ISBN 978-1-4503-4061-8/16/09.. \$15.00

DOI: http://dx.doi.org/10.1145/2967446.2967463

\section{INTRODUCTION}

Bacterial infections are prevalent today and the continued resistance to various types of drugs has led them to emerge as super bugs [19]. The continued and evolved strength against antibiotics is due to their functionalities, which include their ability to interact, signal and communicate, move towards areas of favourable conditions, forming protective structures, as well mutation process that occurs in their DNAs $[8,26]$. In this paper, we will focus on one particular functionality of bacteria that protects and enables them to evolve and resist to antibiotic treatment, and this is known as biofilms.

Biofilms are communities of bacteria that come together, attach to a surface and form a protective medium [8]. The formation of a biofilm begins when bacteria communicate with each other and receive signals from the environment triggering regulatory networks [8] to promote their attachment to a surface with favourable conditions (e.g., sufficient amount of nutrients). After performing the attachment, the signalling process will promote cell division to populate the surface, and production of a protective medium known as the Extracellular Polymeric Substances (EPS) [8]. After creating the EPS, bacteria within this protective medium perform complex communication processes to maintain the biofilm (e.g., division of labor) [10]. Protected by the EPS, the biofilm is hard to penetrate, leading the bacteria to evolve and survive in harsh environments.

Numerous chemical treatments have been developed to treat biofilms [26]. Those treatments will usually lead to dispersal of biofilms $[4,26]$. Another solutions includes jamming the communication process between the bacteria to prevent them to signal in order to create EPS [11]. Also, probiotic bacteria have been used to treat certain biofilmrelated diseases. For example, patients with bowel inflammation and oral mucositis have been subjected to bacteriabased treatments $[13,15]$. In this paper, we propose an alternative mechanism that will disperse biofilms using molecular communication to induce a nutrient competition among different bacterial species. Molecular communication is a new paradigm where the communication system is constructed to represent the signalling process among cells $[2,6]$. This new area of research also extends towards developing artificial communication systems $[1,3,6]$.

The contributions of this paper include: 


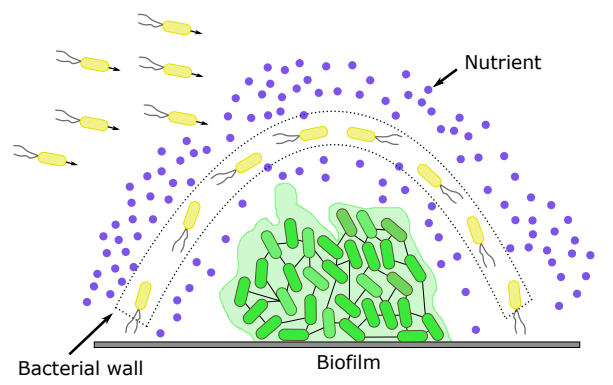

Figure 1: A bacterial wall is formed around the biofilm inducing a competition for available nutrients. Free-moving bacteria are attracted to the wall if their distance from the bacterial wall were smaller than the action radius of quorum sensing molecules.

- Design of biocompatible technique to disperse biofilms: The process concentrates on the natural signalling process between bacteria to induce competing behaviour. The bacteria swim towards an established biofilm, surrounds it and consume nutrients within the environment leading to biofilm starvation (the implicit objective is to trigger social competition within the biofilm that will lead to conflicts and breakage of the biofilm).

- Simulation evaluation: Simulation scenarios are proposed to show the effectiveness of breaking the biofilm through a population of bacteria that surrounds and intercepts the nutrients flowing towards the biofilm.

This paper is organised as follows. In Section 2, the biofilm dispersal process is characterized. The proposed system is formally characterized in Section 3. Section 4 presents the analysis and simulation of the bacterial competition that will lead to the breakage of the biofilm. Lastly, Section 5 presents the conclusion.

\section{BIOFILM AND BREAKDOWN APPROACHES}

Within the human body, biofilms can be formed between the edges of tissues that contain implantable devices [7]. After the biofilms are matured, the EPS protects the bacteria from physical attacks and drug treatments such as antibiotics. Bacteria inside biofilms constantly monitor the environment and induce specific phenotypes as response to any sensed signal [9]. As a response to harsh environments, the dispersal mechanism is activated. This mechanism, commonly used to assure community survival, can also be used to reach other surfaces and spread the biofilm further [19]. In certain cases, these dispersed cells (biofilm flocks or freemoving bacteria) are as harmful as the biofilm itself. For example, in air pipes, biofilm flocks can be carried by the air which can affect people who inhale them [7]. At the same time, biofilms in water pipes must be constantly monitored because they develop a high resistance to chlorine used to treat the water, resulting in high levels of contamination [7].

The current pharmaceutical products are capable of treating biofilms at the early formation stages [26]. However, drugs do not have the same efficiency to kill bacteria inside mature biofilms and, even worse, can promote the development of drug resistance genes. China have recently reported the emergence of "superbugs" that are resistant to all known antibiotics [14]. Therefore, numerous research efforts have been put into mechanisms of dispersing biofilms as a preventive measure for the superbugs $[4,16]$.

Current techniques to induce biofilm dispersal applies quorum sensing molecules and the use of nano particles. Researchers have investigated how disrupting quorum sensing could be used to disperse biofilms and have applied this technique towards the development of new antibiotics [4, 12]. Others are studying how to use nanoparticles as antimicrobials, as they can be lethal to free-moving bacteria [5]. Nanoparticles, as silver ions, can penetrate into biofilms and bond to EPS molecules disrupting it [25]. Although these novel approaches can break biofilms, they require an application of drugs or foreign materials which is not suitable for application in humans since they can result in harmful side effects. For example, nanoparticles need to be precisely injected close to the biofilm, and must not accumulate in specific areas of the tissue due to their high toxicity [21].

An alternative approach is proposed in this paper (see Figure 1). The basic idea is introduce a harsh environment by reducing the amount of available nutrients in order to affect the biofilm's capacity of maintaining the structure. The objective is to utilise bio-compatible mechanism of competing free-moving bacteria to hijack the nutrients. This will prevent the flow of nutrients into the biofilm and disrupt their upkeep leading to the breakage. The paper will present numerical evaluations to demonstrate how the blockage of consumed nutrients by free-moving bacteria can induce biofilm dispersal.

\section{SYSTEM MODEL}

We propose in this paper an induced ecological competition among engineered free-moving bacteria and biofilms. The competition analysis consist of two steps, which are illustrated in Figure 2. The first step consists of sensing biofilm within the environment. Engineered free-moving bacteria will detect a concentration level of QS molecules emitted by the biofilm, and terminate their movement once these levels indicate their proximity to the biofilm in order to form the protective wall. The free-moving bacteria will emit more QS molecules to attract other bacteria within the vicinity to increase the size as well as strengthen the wall. The biased movement results from the activation of their regulatory network once they sense a minimum level of QS molecules [18].

The second step is the nutrient consumption phase by the free-moving bacteria that results in the wall's growth. Due to the number of bacteria within the wall, after a certain period the nutrient consumption rate will be greater than the nutrient renewal process. Therefore, higher efficient nutrient consumption results in increased efficiency to disperse biofilms.

To analyse this process, we model the attraction of freemoving bacteria towards the biofilm to create the wall as a single event (see Subsection 3.1). In Subsection 3.2 we present the growth rate and nutrient consumption rate models for both biofilms and free-moving bacteria.

\subsection{Attraction Model}

To evaluate the attraction process of the free-moving bacteria to the wall we need to obtain the concentration levels of quorum sensing molecules $\left(A_{c}\right)$ that are produced by the 


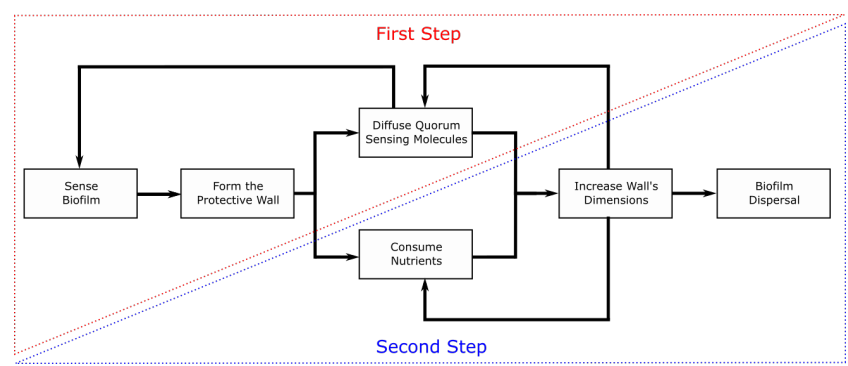

Figure 2: Flow diagram of proposed system. Freemoving bacteria are attracted to the wall and unbalancing the nutrient competition towards them and inducing biofilm to disperse.

wall. These levels can be evaluated as [20, 23]

$$
A_{c}=\frac{D^{2} N_{b a c} r_{v} \beta}{(1+D)\left(1+D+D N_{b a c} r_{v}\right)(\alpha+\beta)},
$$

where $D$ is the diffusion coefficient, $N_{b a c}$ is the number of bacteria, $r_{v}$ is the ratio of cell volume and the volume external to the cell, and $\alpha$ and $\beta$ are the transcription rates. These molecules will be diffused into the environment and will decay exponentially with the distance from the origin,

$$
A_{D}=A_{c} \exp \left(-\sqrt{\left(x-x_{0}\right)^{2}+\left(y-y_{0}\right)^{2}}\right),
$$

where $A_{D}$ is the autoinducer concentration at position $(x, y)$ and $\left(x_{0}, y_{0}\right)$ are the biofilm coordinates.

Considering the $A_{c}$ as the maximum QS level, it is possible to specify the maximum action radius of the protective wall [9]. The action radius is the maximum distance that a bacterium can be with respect to the protective wall, in order for the attraction process to occur. This can be represented as follows

$$
R_{Q S}=\sqrt[3]{\frac{3 c_{A w} D d t}{4 \pi D d A_{c}-c_{A w}}}
$$

where $R_{Q S}$ is the maximum action radius; $d$ is the maximum distance achieved by the diffused molecules; $c_{A w}$ is the transcription basal level for $A H L$ produced by free-moving bacteria at the wall and $t$ is the diffusion time.

\subsection{Growth Rate and Nutrient Consumption Rate Models}

Mathematical models were used to study the effect of freemoving bacteria consumption of nutrients and its effect on the biofilm growth. For the free-moving bacteria, the nutrient consumption and biomass growth rate are represented by (4) and (5) [22]:

$$
\begin{gathered}
\frac{d\left[S_{b a c}\right]}{d t}=-U_{1}\left(\mu_{1} \frac{\left[S_{b a c}\right]}{\left[S_{b a c}\right]+K_{S 1}}\right)\left[N_{1}\right] \\
\frac{d\left[N_{1}\right]}{d t}=\left(\mu_{1} \frac{\left[S_{b a c}\right]}{\left[S_{b a c}\right]+K_{S 1}}-m_{1}\right)\left[N_{1}\right]
\end{gathered}
$$

where $\left[S_{b a c}\right]$ is the nutrient consumption by the bacteria; $\left[N_{1}\right]$ is the bacterial concentration; $\mu_{1}$ is the maximum growth rate; $K_{S 1}$ is the half-saturation constant for free-moving bacteria; $m_{1}$ is the maintenance rate of bacteria cells and $U_{1}$ is an utility parameter.

For the biofilms, other parameters are used to represent the growth and nutrient consumption. Quorum sensing system and EPS production have important roles in biofilm growth, and is highly related to the nutrient consumption rate. For the quorum sensing process, we considered the $\mathrm{N}$ acyl-L-homoserine lactone $(A H L)$ molecules and the $L u x R$ receptors, and their rates are described by (6)-(9) [17]

$$
\begin{gathered}
\frac{d[A]}{d t}=c_{A}+\frac{k_{A}[C]}{K_{A}+[C]}-k_{0}[A]-k_{1}[R][A]+k_{2}[R A] \\
\frac{d[R]}{d t}=c_{R}+\frac{k_{R}[C]}{K_{R}+[C]}-k_{3}[A]-k_{1}[R][A]+k_{2}[R A] \\
\frac{d[R A]}{d t}=k_{1}[R][A]-k_{2}[R A]-2 k_{4}[R A]^{2}+2 k_{5}[C] \\
\frac{d[C]}{d t}=k_{4}[R A]^{2}+k_{5}[C]
\end{gathered}
$$

where $[A],[R],[R A],[C]$ are the $A H L, L u x R, L u x R-A H L$ complex and dimerized complex concentration, respectively. The EPS production that results from the quorum sensing process is represented as [24]

$$
\frac{d[E P S]}{d t}=k_{E P S} \frac{[C]}{[C]+K_{C}} .
$$

The rate expression for the biofilm growth $N_{2}$, considering that bacteria has a limited growth and fixed maintenance rate (this is related to the nutrient consumption when bacteria is neither growing nor dividing) is represented as

$$
\frac{d\left[N_{2}\right]}{d t}=\left(\mu_{2} \frac{\left[S_{b i o}\right]}{\left[S_{b i o}\right]+K_{S 2}}-m_{2}\right)\left[N_{2}\right] .
$$

The nutrient consumption rate $\left[S_{\text {bio }}\right]$ function which is the weighted sum of all rate functions of this process $[22,24]$ is represented as follows

$$
\begin{aligned}
\frac{d\left[S_{b i o}\right]}{d t} & =-U_{2}\left(\mu_{2} \frac{\left[S_{b i o}\right]}{K_{S 2}+\left[S_{b i o}\right]}\right) N_{2}-U_{A H L} \frac{d[A]}{d t}- \\
& -U_{L u x R} \frac{d[R]}{d t}-U_{E P S} \frac{d[E P S]}{d t}
\end{aligned}
$$

where $c_{A}$ and $c_{R}$ are the transcription basal levels for $A H L$ and $L u x R$, respectively; $k_{A}$ and $k_{R}$ are the transcription rates; $K_{A}$ and $K_{R}$ are the degradation rates; $\mu_{2}$ are the maximum specific growth rate for the free-moving as well as bacteria inside the biofilm; $k_{0}, k_{1}, k_{2}, k_{3}, k_{4}, k_{5}$ are the translation rates; $K_{S 2}$ is the half-saturation constant for the biofilm; $m_{2}$ is the maintenance rate; $k_{E P S}$ is the maximum EPS production rate; $K_{C}$ is the dimerized complex degradation rate and $U_{2}, U_{A H L}, U_{L u x R}, U_{E P S}$ are the utility parameters.

Considering that free-moving bacteria and biofilms are coexisting and competing for the same nutrients $[S],(5),(11)$ and (12) are modified as follows, 


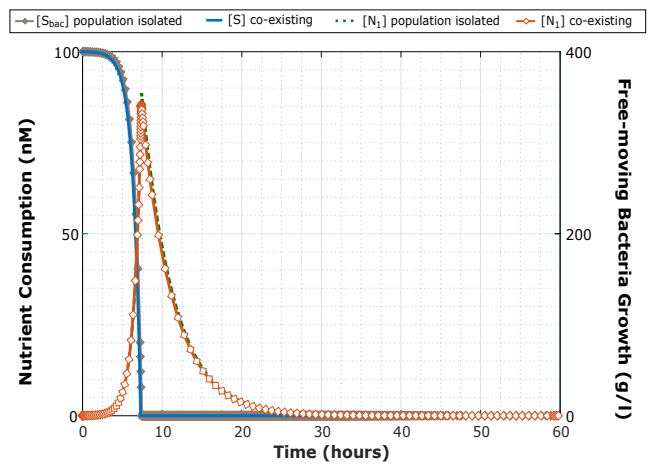

Figure 3: Numerical results of free-moving bacteria nutrient consumption and growth rate when isolated ( $\left[S_{b a c}\right]$ and $\left[N_{1}\right]$, respectively) and co-existing with the biofilm ( $[S]$ and $\left[N_{1}\right]$, respectively).

$$
\begin{gathered}
\frac{d\left[N_{1}\right]}{d t}=\left(\mu_{1} \frac{[S]}{[S]+K_{S 1}}-m_{1}\right)\left[N_{1}\right] \\
\frac{d\left[N_{2}\right]}{d t}=\left(\mu_{2} \frac{[S]}{[S]+K_{S 2}}-m_{2}\right)\left[N_{2}\right] \\
\frac{d[S]}{d t}=-U_{1}\left(\mu_{1} \frac{[S]}{K_{S 1}+[S]}\right)\left[N_{1}\right]- \\
-U_{2}\left(\mu_{2} \frac{[S]}{K_{S 2}+[S]}\right) N_{2}-U_{A H L} \frac{d[A]}{d t}- \\
-U_{L u x R} \frac{d[R]}{d t}-U_{E P S} \frac{d[E P S]}{d t} .
\end{gathered}
$$

The growth rate of free-moving bacteria attracted to the wall $[\mathrm{Ne}]$ is

$$
\frac{d[N e]}{d t}=\mu_{w}[N e],
$$

where $\mu_{w}$ is the rate of free-moving bacteria that is attracted to the wall per hour.

Therefore, both (5) and (15) will be modified to consider (16), and is represented as follows

$$
\begin{gathered}
\frac{d\left[N_{1}\right]}{d t}=\left(\mu_{1} \frac{[S]}{[S]+K_{S 1}}-m_{1}\right)\left(\left[N_{1}\right][N e]\right) \\
\frac{d[S]}{d t}=-U_{1}\left(\mu_{1} \frac{[S]}{K_{S 1}+[S]}\right)\left(\left[N_{1}\right][N e]\right)- \\
-U_{2}\left(\mu_{2} \frac{[S]}{K_{S 2}+[S]}\right) N_{2}-U_{A H L} \frac{d[A]}{d t}- \\
-U_{L u x R} \frac{d[R]}{d t}-U_{E P S} \frac{d[E P S]}{d t} .
\end{gathered}
$$

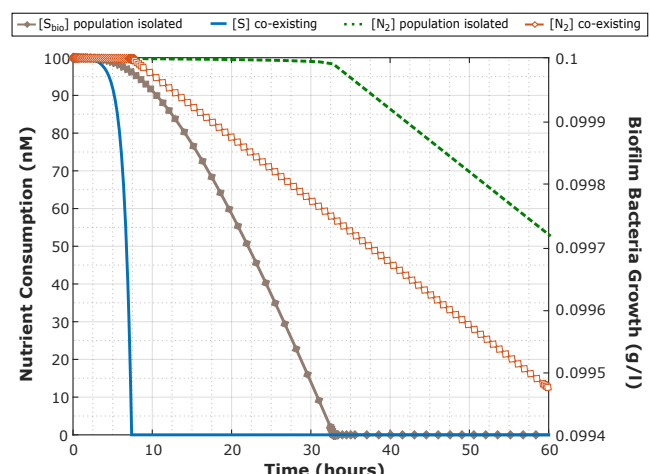

Figure 4: Numerical results of biofilm nutrient consumption and growth rate when isolated ( $\left[S_{b i o}\right]$ and $\left[N_{2}\right]$, respectively) and co-existing with free-moving bacteria ([S] and $\left[N_{2}\right]$, respectively).

\section{BIOFILM DISPERSAL}

In this section, we present the analysis of the biofilm's breakdown mechanism due to the starvation process. All equations shown in Section 3 are solved using the parameters presented in Table 1 . The nutrient consumption rate, biofilm and free-moving bacteria growth rate are required to understand the biofilm's breaking process.

\subsection{Numerical Results}

We analysed the bacterial growth and nutrient consumption rates for the free-moving bacteria and biofilm when they are isolated as well as mixed (forming the wall) in the same environment using (4)-(15). The population of the freemoving bacteria, when isolated, will grow and consume the nutrients as shown in Figure 3. After growing for 7.4 hours, those free-moving bacteria will die due to insufficient nutrients. Figure 3 also shows that they have the same behaviour when they co-exists with the biofilm.

Figure 4 presents the biofilm growth rate over time. Nutrient concentration decays non-linearly over time towards zero producing a fast decaying process for the biofilm after 33 hours when isolated. However, when co-existing with free-moving bacteria, the biofilm decays with a higher rate than when isolated. Its take 10 hours to deplete the nutrients and start the decaying process. From Figures 3 and 4 we can observe that the decay rate of the biofilm is increased by the competition with the free-moving bacteria. Therefore, in order to quickly disperse the biofilm, it is necessary that most of the free-moving bacteria are attracted to the wall within 7.4 hours (this is of course with respect to the quantity of nutrients we have considered).

\subsection{Simulation Results}

Since our objective is to attract most of the free-moving bacteria from the environment towards the biofilm location, we simulated a scenario to determine the quantity of bacteria that will successfully arrive at that location within 7.4 hours. We placed one biofilm at a random position in a closed area $(1000 \times 1000 \mu \mathrm{m})$, with 1000 free-moving bacteria swimming in the area. On average, $53 \%$ of free-moving bacteria were attracted to the wall. We observed the attraction probability over time (five time steps from 1 to 5 hours) to evaluate the rate of free-moving bacteria that will 


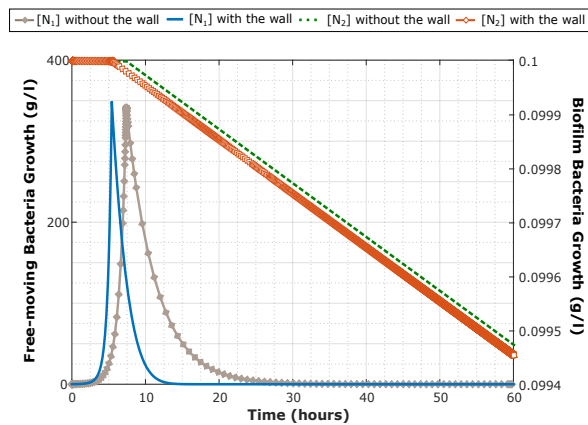

Figure 5: Comparison between free-moving bacteria and biofilm growth rate when co-existing $\left(\left[N_{1}\right]\right.$ and $\left[N_{2}\right]$, respectively) within the same environment, and with the presence of the bacterial wall ([N $\left[N_{1}\right]$ and $\left[N_{2}\right]$, respectively).

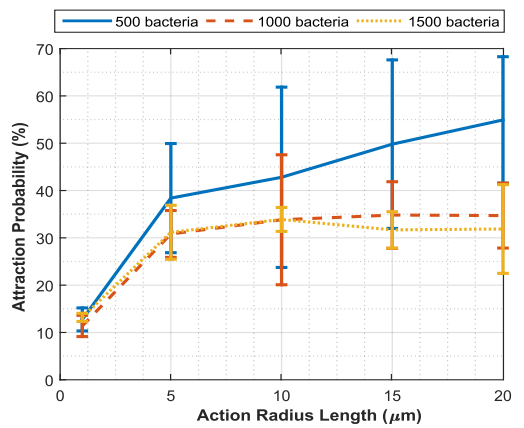

Figure 6: Simulation results for 5 different action radius lengths and three free-moving bacteria numbers (500, 1000, 1500).

continuously enter into the wall. Once the attraction probability of the five time steps are calculated, we used linear regression to obtain the rate of bacteria attraction to the wall $\mu_{w}=0.11$, and this was used to evaluate $(16)-(18)$. The results of integrating the rate of bacteria attraction is illustrated in Figure 5. As we can see from the figure, with continuous addition of free-moving bacteria to the wall, the biofilm started to decay in $\mathbf{5 . 4}$ hours making the natural competition process for the nutrients more efficient.

Figure 6 evaluates the attraction probability when the action radius is varied as well as the number of free-moving bacteria. Once again the biofilm was placed in a random position in a closed area of size $1000 \times 1000 \mu \mathrm{m}$. The $R_{Q S}$ is varied between 1 and $20 \mu \mathrm{m}$ and the number of free-moving bacteria is varied between 500, 1000 and 1500 . The total simulation period is set at 1 hour. As shown in Figure 6, the attraction of free-moving bacteria increases accordingly to that action radius for all considered bacteria number. For 1000 and 1500 , the system achieved its maximum attraction at $R_{Q S}=15 \mu \mathrm{m}$. However, the same does not occur for a few number of bacteria (e.g. 500) in which case a wider action radius is needed.

\section{CONCLUSION}

Over the years, researchers have been developing new solutions that can treat biofilms. The formation of the biofilm results from the bacteria's cooperative behaviour, and once
Table 1: Parameters used to evaluate Eq. (1)-(18)

\begin{tabular}{|c|c|c|}
\hline Variable & Value & Unit \\
\hline$c_{A}$ & $2.7 \times 10^{-2}$ & $\mathrm{nM}$ \\
\hline$c_{A w}$ & $2.7 \times 10^{-2}$ & $\mathrm{nM}$ \\
\hline$c_{R}$ & $2.7 \times 10^{-2}$ & $\mathrm{nM}$ \\
\hline$k_{A}$ & $2 \times 10^{-3}$ & $d^{-1}$ \\
\hline$k_{R}$ & $2 \times 10^{-3}$ & $\mathrm{~d}^{-1}$ \\
\hline$k_{0}$ & $1 \times 10^{-2}$ & $\mathrm{~d}^{-1}$ \\
\hline$k_{1}$ & 0.1 & $d^{-1}$ \\
\hline$k_{2}$ & 0.1 & $d^{-1}$ \\
\hline$k_{3}$ & $1 \times 10^{-2}$ & $d^{-1}$ \\
\hline$k_{4}$ & 0.1 & $d^{-1}$ \\
\hline$k_{5}$ & 0.1 & $d^{-1}$ \\
\hline$k_{E P S}$ & 1 & $\mathrm{~d}^{-1}$ \\
\hline$K_{A}$ & $2 \times 10^{-3}$ & $\mathrm{gm}^{-3}$ \\
\hline$K_{R}$ & $2 \times 10^{-3}$ & $\mathrm{gm}^{-3}$ \\
\hline$K_{C}$ & 1 & $\mathrm{gm}^{-3}$ \\
\hline$K_{S}$ & 1 & $\mathrm{gm}^{-3}$ \\
\hline$\mu_{\max }$ & $1 \times 10^{-4}$ & $\mathrm{gm}^{-3}$ \\
\hline$m$ & $1 \times 10^{-4}$ & $\mathrm{gm}^{-3}$ \\
\hline$U_{X}$ & 0.6 & - \\
\hline$U_{A H L}$ & $2 \times 10^{-2}$ & - \\
\hline$U_{E P S}$ & $2 \times 10^{-2}$ & - \\
\hline$\gamma$ & 0.23 & - \\
\hline$r$ & 1.35 & $\mathrm{~h}^{-1}$ \\
\hline$N_{b a c}$ & from 1000 to 10000 & - \\
\hline$a$ & 0.004 & $\mathrm{gm}^{-3}$ \\
\hline$m_{B}$ & 0.25 & $\mathrm{gm}^{-3}$ \\
\hline$D$ & from 100 to 1000 & $\mathrm{~m}^{2} \mathrm{~d}^{-1}$ \\
\hline$\alpha$ & 6.93 & $\mathrm{nM} /\left(\mathrm{gm}^{-3} \mathrm{~d}^{-1}\right)$ \\
\hline$\beta$ & 2.93 & $\mathrm{nM} /\left(\mathrm{gm}^{-3} \mathrm{~d}^{-1}\right)$ \\
\hline
\end{tabular}

they are formed will create a protective shield that makes them hard to kill. This can lead to infections within the human body as well as contamination within the environment (e.g., biofilms formed in water). Although solutions have been proposed to break and disperse the biofilms, they are based on using nanoparticles or drugs. This paper proposes the use of engineered free-moving bacteria that will cooperate and surround biofilms to hijack the nutrients within the environment. The consumption of the nutrients by freemoving bacteria will lead to minimum nutrients flowing into the biofilm leading to its breakdown. Our numerical analysis has shown how a certain quantity of bacteria that consume the nutrients can grow and populate, and at the same time the starvation of the biofilm can lead to negative growth. Our simulation have also shown that through the cooperative communication of autoinducers by the free-moving bacteria, this can lead to an attraction process that draws in a certain quantity of the microbes towards the biolfim. The proposed approach has shown the promise of using synthetic biology to engineer bacteria that can be used to breakdown and disperse biofilms.

\section{ACKNOWLEDGMENT}

This work has received support from the Science Foundation Ireland (SFI) via the CONNECT research centre under Grant 13/RC/2077, as well as the Academy of Finland FiDiPro program for the project "Nanocommunications Networks" 2012-2016, and the Finnish Academy Research Fel- 
low program under Project no. 284531. This work has also been supported by EU funding from the European Union's Horizon 2020 research and innovation programme under the grant agreement No. 665564 .

\section{REFERENCES}

[1] I. Akyildiz, M. Pierobon, S. Balasubramaniam, and Y. Koucheryavy. The internet of Bio-Nano things. IEEE Communications Magazine, 53(3):32-40, Mar 2015.

[2] I. F. Akyildiz, F. Brunetti, and C. Blázquez. Nanonetworks: A new communication paradigm. Computer Networks, 52(12):2260-2279, Aug 2008.

[3] S. Balasubramaniam and P. Lio'. Multi-Hop Conjugation Based Bacteria Nanonetworks. IEEE Transactions on NanoBioscience, 12(1):47-59, Mar 2013.

[4] N. Barraud, M. J. Kelso, S. A. Rice, and S. Kjelleberg. Nitric Oxide: A Key Mediator of Biofilm Dispersal with Applications in Infectious Diseases. Current Pharmaceutical Design, 21:31-42, Jan 2015.

[5] O. Choi, C. P. Yu, G. Esteban Fernández, and Z. Hu. Interactions of nanosilver with Escherichia coli cells in planktonic and biofilm cultures. Water Research, 44(20):6095-6103, Dec 2010.

[6] L. C. Cobo and I. F. Akyildiz. Bacteria-based communication in nanonetworks. Nano Communication Networks, 1(4):244-256, Dec 2010.

[7] J. W. Costerton and P. S. Stewart. Battling biofilms. Scientific American, 285(1):74-81, Jul 2001.

[8] C. de la Fuente-Núñez, F. Reffuveille, L. Fernández, and R. E. Hancock. Bacterial biofilm development as a multicellular adaptation: antibiotic resistance and new therapeutic strategies. Current Opinion in Microbiology, 16(5):580-589, 2013.

[9] S. J. Hagen. Swimming in Information? Physical Limits to Learning by Quorum Sensing. In S. J. Hagen, editor, The Physical Basis of Bacterial Quorum Communication, Biological and Medical Physics, Biomedical Engineering, chapter 7, pages 123-144. Springer New York, New York, NY, 2015.

[10] M. Hasan, E. Hossain, S. Balasubramaniam, and Y. Koucheryavy. Social behavior in bacterial nanonetworks: Challenges and opportunities. IEEE Network, 29(1):26-34, Jan 2015.

[11] M. Kalia, V. K. Yadav, P. K. Singh, D. Sharma, H. Pandey, S. S. Narvi, and V. Agarwal. Effect of Cinnamon Oil on Quorum Sensing-Controlled Virulence Factors and Biofilm Formation in Pseudomonas aeruginosa. PLOS ONE, 10(8):e0135495, Aug 2015.

[12] A. Kubacka, M. S. Diez, D. Rojo, R. Bargiela, S. Ciordia, I. Zapico, J. P. Albar, C. Barbas, V. a. P. Martins dos Santos, M. Fernández-García, and M. Ferrer. Understanding the antimicrobial mechanism of TiO2-based nanocomposite films in a pathogenic bacterium. Scientific Reports, 4:4134, Feb 2014.

[13] S. A. Limaye, R. I. Haddad, F. Cilli, S. T. Sonis, A. D. Colevas, M. T. Brennan, K. S. Hu, and B. A. Murphy. Phase $1 \mathrm{~b}$, multicenter, single blinded, placebo-controlled, sequential dose escalation study to assess the safety and tolerability of topically applied AG013 in subjects with locally advanced head and neck cancer receiving induction chemotherapy. Cancer, 119(24):4268-4276, Sep 2013.

[14] Y. Y. Liu, Y. Wang, T. R. Walsh, L. X. Yi, R. Zhang, J. Spencer, Y. Doi, G. Tian, B. Dong, X. Huang, L. F. Yu, D. Gu, H. Ren, X. Chen, L. Lv, D. He, H. Zhou, Z. Liang, J. H. Liu, and J. Shen. Emergence of plasmid-mediated colistin resistance mechanism MCR-1 in animals and human beings in China: A microbiological and molecular biological study. The Lancet Infectious Diseases, 16(2):161-168, Feb 2016.

[15] R. Martín, S. Miquel, J. Ulmer, N. Kechaou, P. Langella, and L. G. Bermúdez-Humarán. Role of commensal and probiotic bacteria in human health: a focus on inflammatory bowel disease. Microbial cell factories, 12:71, Jul 2013.

[16] D. McDougald, S. a. Rice, N. Barraud, P. D. Steinberg, and S. Kjelleberg. Should we stay or should we go: mechanisms and ecological consequences for biofilm dispersal. Nature Reviews Microbiology, 10(1):39-50, Jan 2012.

[17] P. Melke, P. Sahlin, A. Levchenko, and H. Jönsson. A cell-based model for quorum sensing in heterogeneous bacterial colonies. PLoS Computational Biology, 6(6):1-13, Jun 2010.

[18] J. G. Mitchell and K. Kogure. Bacterial motility: Links to the environment and a driving force for microbial physics. FEMS Microbiology Ecology, 55(1):3-16, Sep 2006.

[19] N. M. Oliveira, E. Martinez-Garcia, J. Xavier, W. M. Durham, R. Kolter, W. Kim, and K. R. Foster. Biofilm Formation As a Response to Ecological Competition. PLOS Biology, 13(7):e1002191, Jul 2015.

[20] A. Pai and L. You. Optimal tuning of bacterial sensing potential. Molecular systems biology, 5(286):211-286, May 2009.

[21] P. Ramesh and K. Gurunathan. Nanomaterials communication inside the living organism. Nano Communication Networks, 3(4):252-256, Dec 2012.

[22] D. Tilman. Tests of Resource Competition Theory Using Four Species of Lake Michigan Algae. Ecology, 62:802-815, Jun 1981.

[23] M. Weber and J. Buceta. Noise regulation by quorum sensing in low mRNA copy number systems. $B M C$ systems biology, 5(1):11, Jan 2011.

[24] G. Wei, C. Walsh, I. Cazan, and R. Marculescu. Molecular tweeting. In Proceedings of the 6th ACM Conference on Bioinformatics, Computational Biology and Health Informatics - BCB '15, pages 366-375, New York, New York, USA, Sep 2015. ACM Press.

[25] S. M. Wirth, G. V. Lowry, and R. D. Tilton. Natural organic matter alters biofilm tolerance to silver nanoparticles and dissolved silver. Environmental Science and Technology, 46(22):12687-12696, Oct 2012.

[26] H. Wu, C. Moser, H.-Z. Wang, N. Høiby, and Z.-J. Song. Strategies for combating bacterial biofilm infections. International Journal of Oral Science, 7(1):1-7, Dec 2014. 